this should provide an additional stimulus to the development of rapid oligonucleotide synthesis methods.

Several examples of the application of oligonucleotide site-directed mutagenesis are available. It has been used to resolve the functions of two overlapping spliced adenovirus mRNAs made early in infection - a question which could not have been answered unequivocally by random or target-directed mutagenesis ${ }^{14}$. At least two groups have used the procedure to alter the anticodon of a cloned tRNA gene (for example, tRNA ${ }^{\text {Lys }}$ or tRNA ${ }^{\text {Tyr }}$ ) to that of a suppressor tRNA gene. The function of the tRNA was then tested by its ability to suppress various amber mutations (see, for example, ref.15). Finally, the method has been used to probe the function of the signal peptide present on secreted proteins. Changing the charge of the amino acids at the $\mathrm{NH}_{2}$ terminus of the $E$.coli outer membrane protein affected the ability of the protein to be assembled in the membrane ${ }^{16}$.

Clearly, there are a wide variety of biological problems which will benefit from the application of both target- and site directed in vitro mutagenesis. It is now possible to introduce specific changes into cloned DNA; when coupled with high-level gene expression vectors it should also be possible to assay the effect of these changes in several prokaryotic and eukaryotic systems. For certain proteins, for which there is detailed structural information and a cloned gene available, it is not too fanciful to consider that specific mutations could be introduced to produce molecules with altered or novel activities.

\footnotetext{
1. Shortle, D., DiMaio, D. \& Nathans, D. A. Rev. Genet. 15, 265.294 (1981)

2. Putney, S.D., Benkovic, S.J.\& Schimmel, P.R. Proc. natn. Acad. Sci. U.S.A. 78, 7350-7304 (1981).

3. Jasin, M. \& Schimmel, P. Abstract of the meeting.

4. Shortle, D. \& Nathans, D. Proc. naln. Acad. Sci. U.S.A. 75, 2170-2174 (1978).

5. Hayatsu, H. Prog Nucleic Acid Res. molec. Biol. 16, 75-124 (1976)

6. Shortle, D. et al. Proc. natn. Acad. Sci. U.S.A. 77, $5375-5379(1980)$

7. Everett, R.D. \& Chambon, P. EMBO J. 1, 433.437 (1982)

8. Hearing, P. \& Schenk, T. Abstract of the meeting

9. Weiher, H. \& Schaller, H. Proc natn, Acad Sci U.S.A. 79, $1408-1412$ (1982)

10. Muller, W. et al. J. molec. Biol. 124, 343-358 (1978).

11. Shortle, D. et al. Proc. natn. Acad. Sci. U.S.A. 79, Shortle, D. et al.
1588.1592 (1982).

2. Smith, M \& Gillam, S. Genet. Engng 3, 1-32 (1981)

13. Wallace, R.B. et al. Nucleic Acids Res. 9, 3647-3656 (1981).

14. Montell, C. et al. Nature 295, 380-384 (1982).

15. Temple, G.F et al Nature $296,537.540$ (1982).

16. Inouye, S. et al. Proc. natn. Acad. Sci. U.S.A. 79 34438-3441 (1982)
}

\title{
Crown gall tumours and plant growth regulators - any connection?
}

\section{from Mike Bevan}

CROWN gall tumour cells produced by infection with Agrobacterium tumefaciens can grow in culture in the absence of endogenous auxins such as indole acetic acid (IAA), in contrast to normal plant cells. Transformation takes place when part of the giant $\mathrm{Ti}$, or tumour-inducing, plasmid is stably integrated into the nuclear DNA of the host plant. It is not clear whether the tumorous state is caused by the insertion of T-DNA into plant sequences, or if T-DNA encodes control or structural genes for plant growth regulators. Recently, Lui et al. ${ }^{1}$ presented evidence for the presence of an IAA gene on the Ti plasmid. Although mutations in the IAA locus render the bacteria harbouring the plasmid avirulent, the locus itself is in a part of the plasmid that is not stably maintained in tumours.

The gene, iaaP, responsible for IAA synthesis was identified on the tumourinducing plasmid by inserting the plasmid into an avirulent, plasmid-free mutant that did not synthesize IAA ( $A$. tumefaciens cells themselves normally produce large amounts of IAA). The iaaP locus, which may encode enzyme(s) responsible for the conversion of indolepyruvate to IAA, was

Mike Bevan is in the Plant Breeding Institute, Mavis Lane, Trumpington, Cambridge $C B 22 L Q$. mapped by transposon ( $\operatorname{Tn} 5)$ mutagenesis to a region of the $\mathrm{Ti}$ plasmid known to be involved in oncogenesis. These sequences, called the virulent region, have been delineated by transposon mutagenesis to a region of about 30 kilobases (in pTiB6806 ${ }^{2,3}$ ) which may mean that many traits other than IAA production are involved in virulence. It remains to be demonstrated that this Tn5 insertion does not have polar effects on adjacent virulence genes. The question now remains, how do these virulence genes, in particular the iaaP locus, incite tumour formation when it is only the T-DNA, some $20 \mathrm{~kb}$ from the virulent region, that is stably maintained in the plant tumour?

Lui et al. have three suggestions. First, IAA secreted by virulent bacteria may 'precondition' a wounded plant cell by inciting cell wall changes and DNA synthesis which would allow subsequent transformation. This is reminiscent of gall induction on olive and oleander stems by Pseudomonas savastanoi, which harbours a plasmidencoded enzyme that converts indoleacetamide to IAA. Virulence, as measured by gall formation, is directly related to IAA levels ${ }^{4}$, and no transfer of plasmid DNA to the host plant has been observed. Second, the authors speculate that IAA may be a 'second messenger' that activates other virulence genes when the bacterium encounters a suitable plant cell. Finally, it is suggested that some sort of DNA arrangement may integrate the IAA gene(s) into T-DNA in an early transient stage of crown gall formation.

Recent evidence from extensive transposon mutations in T-DNA indicates that several genes in a highly conserved region of T-DNA are involved in tumour growth and morphology. Two genes, when mutated, give rise to a shooty phenotype, and one gene gives rise to a rooty phenotype when mutated ${ }^{5}$. These findings can be formally explained by postulating that the rooty locus regulates cytokinin levels, and the two shooty loci regulate auxin (IAA?) levels. Ooms et al. ${ }^{6}$ demonstrated that exogenous auxin reversed the shooty phenotype and exogenous cytokinin reversed the rooty phenotype in mutated octopine tumours. Interestingly, nearly all of T-DNA can be deleted without affecting the ability of remnant left and right border fragments to be transferred into plant DNA $^{7}$. These 'transformed' cells are, of course, non-tumorous. The virulence region, which maps outside T-DNA, has been implicated in the transfer of T-DNA to plant cells.

In the light of these findings there may be no need to postulate the integration of the non-T-DNA auxin gene into plant DNA to explain oncogenesis, as Lui et al. suggest. Indeed, the significance of IAA production by Agrobacteria is unclear as in the absence of endogenous tryptophan, both virulent and avirulent mutants failed to accumulate IAA $^{8}$. Perhaps there is adequate tryptophan present in the cell sap of the wound where gall formation is initiated to induce IAA synthesis by virulent Agrobacteria, but this has not been investigated.

What does seem clear is that it is profitable to consider auxin and cytokinin autotrophy, regulated by loci on conserved sequences in T-DNA, as being important, if not the basis, for the autonomous growth of crown gall tumours. Endogenous growth regulator levels in plant cells are known to vary widely, and it is reasonable to assume that both sources of growth regulators contribute to the final growth rate and morphology of tumours. The search for a definite connection between the shooty and rooty loci and growth regulator production has been confounded by the very low concentrations of mRNA and proteins encoded by this region of T-DNA. A promising approach has been to direct the expression of T-DNA-encoded genes in Escherichia coli minicells ${ }^{9}$, and identify the activity of T-DNA-encoded proteins.

\footnotetext{
1. Lui, S.-T., Perry, K.L., Shardl, L.L. \& Kado, C.I Proc. natn. Acad. Sci. U.S.A. 79. 2812-2816 (1982) 2. Garfinkel, D.J. \& Nester, E.W. J. Bact. 144, 732-743 (1980)

3. Ooms, G, Klapwijk, P.M., Poulis, J.A. \& Schilperoort, R.A. J. Bact. 144, 82-91 (1980)

4. Smidt, M.L. \& Kosuge, T. Physiol. Pl. Path. 13, 203-214 (1978).

5. Garfinkel, D.J. et al. Cell 27, 143-153 (1981).

6. Ooms, G., Hooykaas, P.J.J., Moolenaar, G. \& Schilperoort, R.A. Gene 14, 33-50 (1981)

7. Leemans, J. ef al. EMBO J. 1, 147-152 (1982).
} 